\title{
Impact of Environmental Factors on Production and Reproduction in Domestic Animals
}

\author{
Dr.Gurdeep Singh ${ }^{1}$, Dr.Amitoz Kour ${ }^{2}$, Dr.Gyan Prakash Pathak ${ }^{3}$ \\ ${ }^{I}$ Department of Animal Genetics \& Breeding, DGCN COVAS, Palampur, H.P, India, 176062 \\ ${ }^{2}$ Department of Veterinary Microbiology, DGCN, COVAS, Palampur, H.P, India, 176062 \\ ${ }^{3}$ Department of Veterinary Pharmacology and toxicology, DGCN, COVAS, Palampur, H.P, India, 176062
}

\begin{abstract}
Climatic restrictions on vectors, environmental habitats and disease causing agents are important for keeping many animals diseases in check. Alterations of temperature and precipitation regimes may result in a spread of disease and parasites into new regions or produce an increase in the incidence of disease, which, in turn, would reduce animal productivity and possibly increase animal mortality. Some current practices to reduce heat stress in dairy cows, such as shades, sprinklers and ventilation will be suitable for adapting to future climates if the economics of heat stress management do not change radically. However, farmers are not quite aware about the impacts global warming can produce in their operation. Therefore, good research work is needed to help them take strategic and tactical decisions.
\end{abstract}

Key words: Habitats, precipitation, ventilation, global warming and tactical.

\section{Introduction}

Reproduction in farm animals is highly affected by environmental factors and when environmental conditions are favorable, reproductive activity expresses its full potential. Favourable conditions must include adequate photoperiod, thermo-neutral conditions, food availability in quantity and quality and a low stress environment. The body temperature of most domestic animals is considerably higher than the environmental temperature to which they are exposed most of the time, so they maintain their body temperatures by balancing internal heat production and heat loss to the environment. The hypothalamus gland acts as a body thermostat by stimulating mechanisms to counteract either high or low ambient temperatures. Regulation of body temperature under physiological processes involves temp regulation system which covers 3 basic components:

$>$ Sensors involving Peripheral cold \& warm receptors.

$>$ Thermostatic control unit (Bianca, 1968) maintains core body temperature, consisting of heat loss center i.e anterior hypothalamus \& heat production center consisting of post hypothalamus.

> Thermo regulatory effectors involving peripheral vasoconstriction or dilatation, sweating, panting, shivering \& non shivering or endocrine.

\section{Direct impacts on animals}

Folk(1974) demonstrated that the main natural physical environmental factors affecting livestock are air temperature, relative humidity, radiant heat, precipitation, atmospheric pressure, UV-light, wind velocity and dust. Impact of hot climate on animal productivity causes maintenance requirement to decrease and feed intake to decrease, egg production (Anjam et al,2002), egg shell thickness to decrease and wool production to increase. Response of animal to environment changes involves:

$>$ Homeostasis which is a maintenance of constant internal environment despite variation in external environment including (body temperature, body water, blood pressure, ph, ionic environment) and heterothermy which is abilty to vary core temperature and plays significant role in animal adaptation under desert condition.

Thermal balance/ Heat balance: expressed as Heat production $=$ Heat loss + Heat gain + Heat storage Lack of ability of animals to dissipate the environmental heat causes the animal susceptible to thermal/heat stress.

\section{Causes of Heat /thermal stress are}

$>$ Animal's inability to dissipate sufficient heat to maintain homeothermy and homeostasis,

$>$ High ambient temperature, \& relative humidity,

$>$ Radiant energy and

$>$ Increase in air temperature.

Thermal stress impact on reproduction causes

$>$ Reduction in conception rate.

$>$ Embryo mortality (Gwazdauskas et al,1985), 
$>$ Impairs luteal function (Wolfenson et al,1993),

$>$ Disturbs gonadotrophin \& oestradiol secretion(Wilson et al,1998),

$>$ Modifies estrus expression \& follicular dynamics,

$>$ Inc. frequency of silent estrous and

$>$ Development of ovarian cysts.

Thermal stress on milk yield causes

$>$ Linear reduction in milk yield, if env. temp exceeds $22-23^{\circ} \mathrm{c} \&$ Temperature humidity index (THI) exceeds 70 .

$>$ Heat stress causes $35 \%$ dec. in milk yield in mid lactating cows \& $14 \%$ dec in early lactating dairy cows.

$>$ Exposure of lactating ewes to solar radiations under HAT (high ambient temperature) reduces milk yield to $20 \%$. and Lactating goats exposed to moderate or severe hot for 4 days decrease milk yield by $3 \& 13 \%$ respectively

$>$ Water buffaloes in summer have less milk yield.

Thermal stress on health causes increase in parasitic diseases resulting in death of livestock, Metabolic changes, Pro-oxidant antioxidant balance increasing free radicals conc. \& reactive oxygen spp, leads to damage of macromolecules $\&$ disruption of normal metabolism and physiology and changes of immune function.

Environmental management: of animals need to be done at three levels i.e

$>$ Strategic decision which recognize ability of animal to acclimatize for env. Challenges, involving Housing, Shades, Wind breaks, THI as a thermal index (Dairy cattle show signs of heat stress when THI is higher than 72), and if THI $>78$, Cows production decreases and if THI $>82$, then there is loss of milk production.

$>$ Tactical decision: evaluate consequence of current weather, involving use of Sprinklers, Fans, Heaters.

$>$ Developing climatology of summer weather extreme provides knowledge to control.

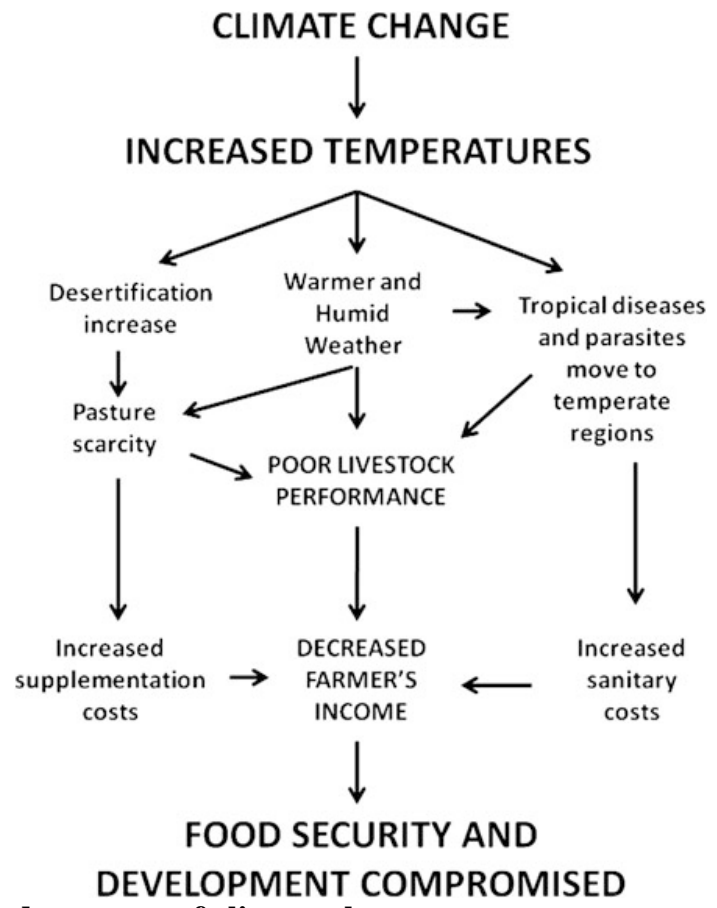

Schematic view of the expected outcome of climate change as a consequence of global warming on farm animal productivity

\section{Conclusion}

The adverse factors in farm animal productivity is essential to design mitigation strategies at the local, regional, national, and transnational level. In our opinion, it is vital that such strategies would focus on the study and use of local genetic resources showing a high level of adaptation to the most significant issue for that specific region, either climate, disease, or nutrition-induced. Such studies have necessarily to be directed towards the full comprehension of the local breed's genetic background and production ability, as well as the 
search for markers of tolerance to those limiting factors. Such markers would be of great importance and use in the definition of selection strategies and objectives to increase livestock productivity, with special reference to developing countries.

\section{Refrences:}

[1] Anjum, MSZ; Rehman, M; Akram, SM; Sandhu, MA. Haemotochemical profile of commercial layers influenced by heat combating systems during high ambient temperature.In:Proc. $15^{\text {th }}$ Conf Biometeorol Aerobiol; 2001:102-103.

[2] Bianca W.Thermoregulation. In:Hafez ESE; Adaptation of domesticated animals,Lea \& Febiger, Philadelphia, USA; 1968: 97-118

[3] Folk, JE.What is environmental physiology: history and terminology. In: Textbook of environmental physiology, Lea \& Febiger, Philadelphia, USA; 1974: 1-16.

[4] Gwazdauskas FC. Effect of climate on reproduction in cattle. J. Dairy Sci; 1985: 68: 1568-1578.

[5] Wilson SJ; Marion RS; Spain JN, Spiers DE; Keisler DH; Lucy MC. Effects of controlled heat stress on ovarian function of dairy cattle: lactating cows. J.Dairy Sci; 1998: 81: 2124-2131.

[6] Wolfenson D; Luft O; Berman A; Meidan R.Effects of season, incubation, temperature and cell age on progesterone \& prostaglandin production in luteal cells. Anim. Reproduction Sci; 1993: 32: 27-40. 